\title{
Analysis of the performance of hydrogen maser clocks at the Hartebeesthoek Radio Astronomy Observatory
}

\author{
C Munghemezulu ${ }^{1,2}$, L Combrinck $^{1,2}$, OJ Botai ${ }^{1,3}$ and J Quick ${ }^{2}$ \\ ${ }^{1}$ Department of Geography, Geoinformatics and Meteorology, Centre for Geoinformation \\ Science, University of Pretoria, South Africa, \\ cilence.munghemezulu@up.ac.za \\ ${ }^{2}$ Hartebeesthoek Radio Astronomy Observatory, P O Box 443, Krugersdorp 1740, South \\ Africa \\ ${ }^{3}$ South African Weather Services, Pretoria, South Africa
}

http://dx.doi.org/10.4314/sajg.v5i3.5

\begin{abstract}
Hydrogen maser frequency standards are commonly utilised in various space geodetic techniques such as Very Long Baseline Interferometry (VLBI) as local reference clocks. The Hartebeesthoek Radio Astronomy Observatory in South Africa is currently operating two maser frequency standards i.e., an EFOS28 and an iMaser72 for the $15 \mathrm{~m}$ and $26 \mathrm{~m}$ VLBI radio telescopes respectively, an older EFOS6 is a standby spare. This study utilised the least-squares method to derive clock parameters, which indicates the performance levels of the masers by making use of the offset measurements obtained between hydrogen maser clock 1 PPS and GNSS 1 PPS for a period of 35 days. The masers were also compared using a frequency comparator (VCH-314) for a time period of $100 \mathrm{~s}$. The results indicate that the performances of both Masers are relatively similar to each other, with short-term and longterm results indicating good agreement. The iMaser 72 has a better standard error of 0.0039 $\mu$ s compared to the standard error of $0.0059 \mu$ s for the EFOS28 maser clock. In general, both masers performed at an expected level required for radio astronomy and geodetic VLBI applications. The method used in this study proved to be useful in managing local hydrogen maser clocks to ensure accurate VLBI observations are obtained.
\end{abstract}

\section{Introduction}

Most geodetic techniques rely on the accuracy and stability of their reference clocks; the clock characteristics have a direct influence on precision and accuracy of the observations. To achieve accurate observations at the sub-centimetre level, the local clocks must be accurate to sub-picoseconds (ps) level. Different timing reference standards are used to provide accurate time in geodetic and astronomical instrumentations such as Caesium, Rubidium, Oven Controlled Crystal Oscillators (OCXO) and atomic hydrogen maser (maser is an acronym for microwave amplification by stimulated emission of radiation) clocks. For the Very Long 
Baseline Interferometry (VLBI) applications, hydrogen maser clocks are recommended as frequency standards (Levine and Vessot 1970; Schuh and Böhm 2012). Other clocks are mainly used in Satellite Laser Ranging (SLR) or Lunar Laser Ranging (LLR) applications. For example, the MOBLAS-6 SLR station at the Hartebeesthoek Radio Astronomy Observatory (HartRAO) utilises a Rubidium-disciplined crystal oscillator for local reference time (Combrinck, 2009). It is well known that clocks drift over time due to thermal instabilities, noise in the electrical circuits, etc. Hence, they need to be adjusted after some time to compensate for drifts and ageing characteristics to allow proper alignment to the Coordinated Universal Time (UTC) to within a few microseconds (Allan, 1987; Lewandowski et al., 1999).

Atomic fountain clocks with high accuracy and stability have been developed to improve the measurement of time with uncertainties of a few parts in $10^{-16}$ (Wynands and Weyers 2005). The National Institute of Standards and Technology (NIST) is currently operating two Ceasium Fountain Clocks (NIST-F1 and NIST-F2) to define time with supreme accuracy (Donley et al., 2004). Optical frequency standards using a spin-1/2 system has been reported to yield better accuracy than the microwave based frequency standards (Lemke et al., 2009). Ely et al., (2014) and Seubert and Ely (2015) have reported on a small mercury-ion atomic clock with Allan deviation of less than $10^{-14}$ per day for use in deep space applications through the Deep Space Atomic Clock (DSAC) programme of NASA. However, these clocks have not yet been employed in VLBI applications but are bound to be used in the future when these clocks become affordable.

Friedrich Robert Helmert (1843-1917) defined geodesy as the science of measurements and mapping of the Earth's surface. This definition has evolved over time due to technological advancements and is now defined as the science of determining the geometry, gravity field and rotation of the Earth and the evolution of these three pillars over time (Plag et al., 2009). Combrinck (2014), proposed a fourth pillar of geodesy i.e., spacetime curvature. The General Theory of Relativity (GRT) describes the spacetime curvature and its effects on all measurements made by space geodetic techniques (Ciufolini, 1990; Combrinck, 2013). Hence, it is important to consider spacetime curvature as one of the pillars of modern geodesy due to its influence on geodetic observations. For instance, clocks in orbit around the Earth run faster than on Earth due to the weaker gravity field, this effect has to be compensated for. Space geodesy makes use of Global Navigation Satellite Systems (GNSS), SLR, VLBI, and Doppler Orbitography and Radiopositioning Integrated by Satellite (DORIS) techniques to study the Earth system; all these techniques have very stringent clock requirements.

The VLBI technique, in particular, is important for studying positions and structural variability of astronomical radio sources (e.g., quasars) by utilising a network of radio telescopes. Even though this technique was originally developed by astronomers for radio astronomy research, it is also applied in geodesy (in an inverse sense) to study geodynamics 
by determining baselines and their rates between different stations (Ronnang, 1989). The technique also defines and maintains the International Celestial Reference Frame (ICRF). The Earth Orientation Parameters (EOP) are also determined with high accuracy and they include polar motion, precession-nutation and Length of Day (LOD). The technique also contributes towards the International Terrestrial Reference Frame (ITRF) by providing positions of the VLBI antennas and the reference frame scale component (Schuh and Böhm 2012). These data products are important for a number of applications such as establishment and maintenance of reference frames and determination of orbit parameters for spacecraft and satellites.

HartRAO operates two VLBI radio telescopes (Figure 1). The telescopes function within the framework of the International VLBI Service for Astrometry and Geodesy (IVS) and participate in various international programmes. They use hydrogen maser clocks as their local reference timing systems. We evaluate and compare the performance of the two hydrogen maser clocks by using the least-squares approach to determine their clock parameters.

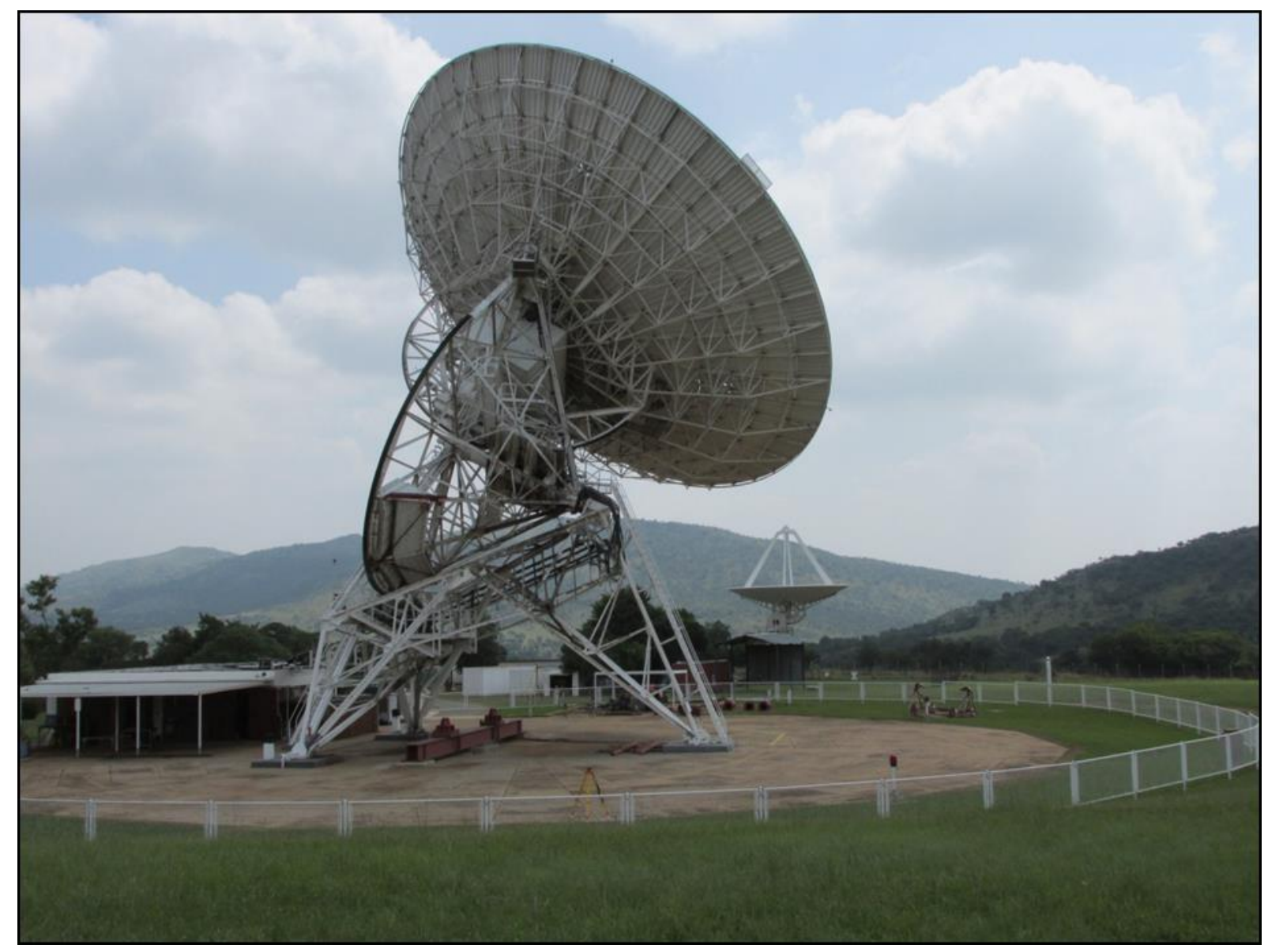

Figure 1. The VLBI radio telescopes located at HartRAO, South Africa. The $26 \mathrm{~m}$ antenna is located in the forefront and the $15 \mathrm{~m}$ antenna can be seen in the background. 


\section{Hydrogen MASER clocks at HartRAO}

The iMaser72, EFOS28 and EFOS6 hydrogen maser clocks used at HartRAO for VLBI applications can be seen in Figure 2. They are stored in a temperature controlled room, which is maintained at $25.7 \pm 0.5^{\circ} \mathrm{C}$. Each maser rests on an independent pillar of the floor foundation, the pillars are also designed with thermal insulators so that they do not influence the temperature stability of the maser enclosure and that of the room. The EFOS28 maser replaced the EFOS6, which was in service for 18 years prior to installation of the EFOS28 maser by the Observatoire Cantonal de Neuchatel in 2003. The EFOS28 has an Allan deviation of $1.5 \times 10^{-13}$ at $1 \mathrm{~s}$ and thermal stability of $1.9 \times 10^{-15} /{ }^{\circ} \mathrm{C}$ as specified by the manufacturer. The iMaser72 was installed in 2013. Its manufacturer specifications are $1.26 \times 10^{-13}$ at $1 \mathrm{~s}$ Allan deviation and it has a thermal stability of $3.5 \times 10^{-15} /{ }^{\circ} \mathrm{C}$.

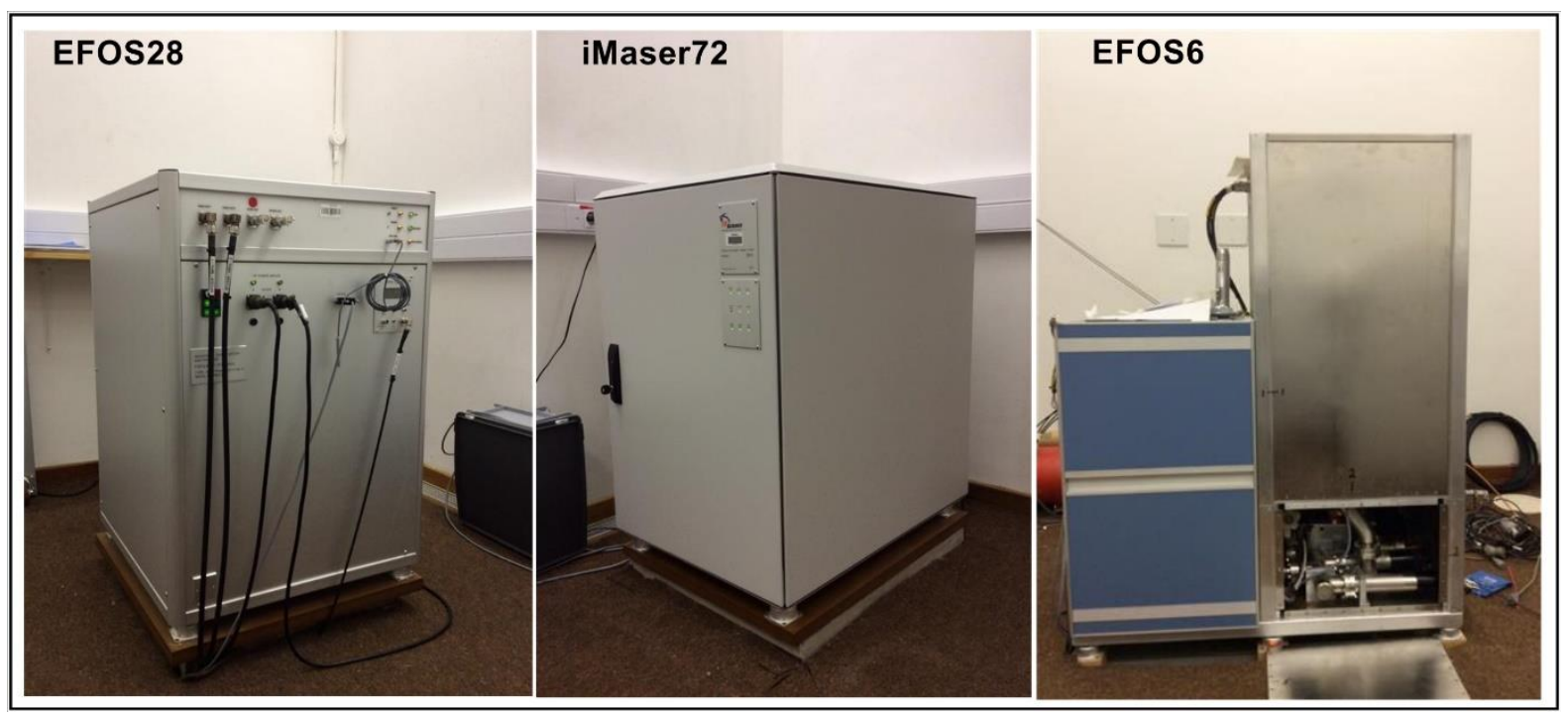

Figure 2. The EFOS28, iMaser72 and EFOS6 hydrogen Maser clocks of HartRAO. The iMaser72 and EFOS28 are utilised as frequency standards for the $26 \mathrm{~m}$ and $15 \mathrm{~m}$ VLBI telescopes respectively; the EFOS6 Maser is for backup purposes.

Hydrogen Maser clocks are based on the resonance frequency of the hydrogen atom $(1420405751.68 \mathrm{~Hz})$. Usually, hydrogen is supplied by heating a solid state metallic compound $\left(\mathrm{LaNi}_{5} \mathrm{H}\right)_{\mathrm{x}}$, which releases the gas. The molecular hydrogen is purified and stored in the discharge bulb. The beam of hydrogen atoms are passed through a multiple magnetic pole state selector ( 4,6 or 8 poles can be used).

Depending on the energy state of the hydrogen atoms, those with upper levels designated by $\mathrm{F}=1, \mathrm{~m}_{\mathrm{f}}=1$ and $\mathrm{m}_{\mathrm{f}}=0$ will be directed towards a quartz storage bulb and those with lower levels (i.e, $\mathrm{F}=0, \mathrm{~m}_{\mathrm{f}}=-1, \mathrm{~m}_{\mathrm{f}}=0$ ) will be pumped off. The quartz storage bulb is coated with Teflon to minimise interaction with the hydrogen atoms. The bulb is stored in a cavity that is thermally and magnetically protected (having a typical shield factor of $10^{5}$ ), and tuned to a 
frequency of $1420 \mathrm{GHz}$ which is related to the upper energy levels of the hydrogen atoms (Vessot, 2005). Atoms in the storage bulb continuously interact and during this process, they lose energy and translate from upper level to lower level state. The photons that are released cause other upper-level atoms to lose energy as well. This results in a self-sustaining microwave field in the bulb. The low maser signal (about $10^{-13} \mathrm{~W}$ ) generated in the cavity is amplified and locked to a resonance frequency of the hydrogen atom and is utilised to generate a one pulse per second (1 PPS) and a standard frequency signal (typically 5 or 10 $\mathrm{MHz}$ ) by the frequency synthesiser system. More information can be found in Lombardi (2002); Mizuhiko (2003); Middelberg and Bach (2008); Vessot (2005); where detailed information about hydrogen maser clocks and the factors that affect their performance levels are discussed.

\section{Basic principles of the VLBI technique}

The VLBI technique is designed to study extra-galactic and radio sources (quasars). Among other applications, VLBI results are applied in determining EOP values as well as expanding and maintaining the ICRF with high angular resolution. The angular resolution of a single antenna dish can be approximated by

$$
\varepsilon \approx \frac{\lambda}{d}
$$

where $\lambda$ is the wavelength of a particular radio source and $d$ is the telescope diameter. To improve the angular resolution, observations can be made through a configuration of a network of antennas that are widely distributed around the world. In this case, the size of the Earth becomes the diameter of one big single antenna dish that can reach an angular resolution of about 1 mas (milli-arcsecond) or better (Seeber 1993, Schuh and Heinkelmann 2010). In the early days of VLBI experiments, the telescopes observing the same source in a network were configured as a Connected Element Interferometer (CEI), where the observing telescopes were connected to a single oscillator by a fibre cable (Edwards et al., 1992). This method limits the baseline length. Today, most telescopes are equipped with atomic hydrogen maser clocks to allow for inter-continental baseline lengths to be achieved.

The observation made by two or (more telescopes) is the difference in arrival time (group delay, $\tau_{g}$ ) of a planar wave signal ( $s_{o}$ being the direction of the source) from quasars or an extragalactic radio source received by the two or more antennas separated by a vector $b$ (Robertson 1991; Figure 3). The group delay can then be defined by the scalar product of $s_{o}$ and $b$ divided by the speed of light $(c)$,

$$
\tau_{g}=\frac{b \times s_{o}}{c}=\left(t_{1}-t_{2}\right)
$$




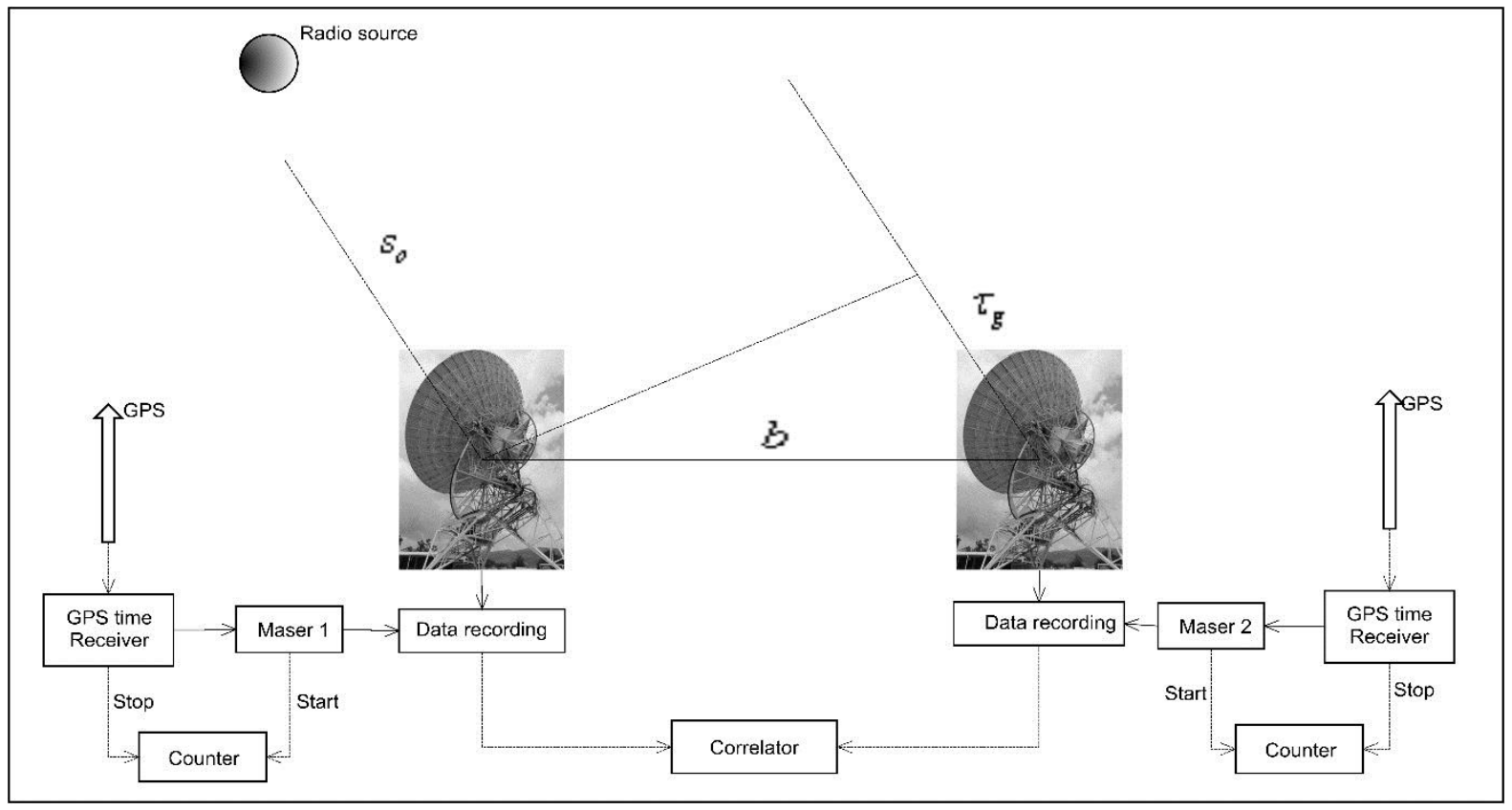

Figure 3. Basic principles of VLBI technique and measurement method for the clock offset data.

A complete observation equation should include corrections for bias and clock drift, atmospheric refraction, special and relativistic effects, cable delay and diurnal aberration. For more details on VLBI, see Seeber (1993); Schuh and Böhm (2012); Schuh and Behrend (2012). During observations, data are recorded on disc packs if the baselines are large and correlated at a later stage. If the telescopes are close to each other, they can be connected via CEI and data can be transmitted to the correlator almost instantly. In recent years, electronic VLBI (e-VLBI) has been developed to allow data correlation of longer baselines via broadband data transmission and real-time data processing.

Existing VLBI telescopes are being upgraded and new telescopes are being developed through the initiatives of the IVS with the aim of achieving $0.1 \mathrm{~mm} / \mathrm{yr}$ and $1 \mathrm{~mm}$ accuracy in reference frame stability and station position respectively (Petrachenko et al., 2009). The new antennas have been named "VLBI2010 Global Observing System" (VGOS) antennas. HartRAO is currently in the process of building one of the VGOS antennas, this new antenna will improve the observations and strengthen the network in the Southern Hemisphere (Mayer et al., 2014). Niell et al., (2005) identified a number of strategies to achieve $1 \mathrm{~mm}$ accuracy and one of them is to improve the frequency reference standards that are used at observatories. To achieve $1 \mathrm{~mm}$ accuracy, the frequency standard accuracy must reach a stability of few parts in $10^{-16}$ for averaging times longer than 1 hour. The frequency stability of about $\pm 5 \times 10^{-13}$ in a 24 hour period is usually required in the current operational requirements (Wei-qun et al., 2001).

Moran (1989) explains further the importance of accurate timing systems in geodetic VLBI and astronomy, where the Signal-to-Noise ratio (SNR) received can be written as 


$$
S N R=\frac{S A \sqrt{2 B \tau_{c}}}{k T_{s}}
$$

In Equation 3, $S$ is the source density flux, $A$ is the geometric mean of the telescope collecting areas, $B$ is the bandwidth, $k$ is Boltzmann's constant, $T_{s}$ is the geometric mean of the system temperature of the telescope and $\tau_{c}$ is the coherent integration time, which can be estimated by

$$
\omega \times \tau_{c} \times \sigma_{y}(T)=1
$$

In Equation 4, $\sigma_{y}(T)$ is the two-sample Allan variation and $\omega$ is the local oscillator frequency in radians per second. For example, to achieve a signal coherence for an observation period of $1000 \mathrm{~s}$, if the local oscillator is set to $8.0 \mathrm{GHz}$, the two observing stations must maintain relative stability of $\sim 2 \times 10^{-14}$ (Combrinck, 2013). Therefore, it is important for observatories to keep track of their hydrogen maser clock performance and to make sure that the drifts and ageing characteristics are properly understood and corrected to allow accurate measurement during experiments.

\section{Data and Analysis Strategies}

\section{1. $\quad$ Long-term data}

Hydrogen maser clocks at VLBI sites require calibration to account for systematic drifts. This requires comparison with an external independent stable timing reference system, which is accomplished through time transfer technique by utilising GNSS technology (Lewandowski et al. 1999). Data from the network of hydrogen maser clocks (U.S Naval Observatory-USNO) are used to update the GNSS satellites with timing information, and then the satellites transmit updated time and navigation messages to the user. The transmitted information is then used to allow local timing systems to be calibrated to the USNO UTC time.

Two hydrogen maser clocks i.e., iMaser72 and EFOS28 are used for the $26 \mathrm{~m}$ and $15 \mathrm{~m}$ telescopes respectively for geodesy and astronomy observations at HartRAO. The two masers are connected to a GNSS time receiver and high-speed counters are used to measure the offsets between 1 PPS (1 Pulse Per Second) from the Maser clocks and from the GNSS UTC time receivers (Figure 3). The maser 1 PPS signal is used as the start and the 1 PPS signal from the GNSS receiver is used as the stop signal. The measurements are made by high-speed counter every second but averaged to a 24 hour period, later on, this is to minimise short-term noise in the GNSS due to ionospheric and tropospheric effects and taking into account the orbit period of satellites, which repeats every 24 hours (Lombardi, 2002). Data were collected simultaneously for the two maser clocks for 35 days starting from 04-15-2015 to 05-19-2015 
period. The data contains daily averaged offset measurements between each maser clock and GPS UTC in $\mu \mathrm{s}$.

The 24-hour offset data sets were analysed by using a second quadratic trend model given by Levine et al., (1999) as

$$
Y_{i}=b_{o}+b_{1} x_{i}+b_{2} x_{i}^{2}
$$

The coefficients for Equation 5 were determined using a least-squares curve fit in Microsoft Excel. The drift parameters of the clocks were calculated by taking the first derivative of Equation 5 to arrive at Equation 6 , where $\Delta t$ is the range period of the observations. The units are given as microseconds per day $(\mu \mathrm{s} / \mathrm{d})$ for Equation 6 . By taking the derivative of Equation 6, ageing parameters of the clocks can be derived in microseconds per day per day $(\mu \mathrm{s} / \mathrm{d} / \mathrm{d})$ as given by Equation 7 ,

$$
\begin{aligned}
& \text { drift }=b_{1}+2 b_{2} \Delta t, \\
& \text { Ageing }=2 b_{2} .
\end{aligned}
$$

The EFOS28 MASER clocks can be adjusted by using Equation 8 , where $f_{H}$ is the Hydrogen MASER frequency $(1402405751.6893 \mathrm{~Hz}), \pm \Delta f$ is the fractional frequency error (calculated by dividing the drift by the number of seconds in a day) and this value can be positive or negative depending on the behaviour of the MASER, and $f_{\text {synth }}$ (this is the last value obtained when correcting the maser, in this case, we use the initial value of 5751.68267),

$$
f_{a d j}(E F O S 28)=f_{\text {synth }}-\Delta f \times f_{H} .
$$

The iMaser72 clock is adjusted by using Equation 8, where the fractional frequency error is either added or subtracted in the Direct Digital Synthesizer (DDS) value, (in this case, the initial DDS value for the iMaser72 is 1420405750.291886) and the maser software will carry out further computations to adjust the output frequency of the maser and determine the new DDS value based on the input $\pm \Delta f$,

$$
f_{a d j}(i M A S E R 72)= \pm \Delta f \text {. }
$$

It is important to note that the adjustment procedure for different maser clocks will be different depending on the design and the manufacturer. 


\subsection{Short-term data}

The short-term stability variations were analysed using a frequency comparator (VCH314). The three oscillators, two-channel model (Chernyshev et al., 2012) was used to measure frequency instabilities of the iMaser72, EFOS28 and EFOS6 masers simultaneously. The EFOS6 maser was used as a reference for the other two maser clocks. This particular setup reduces systematic errors due to instabilities of the reference clock and comparator (Groslambert et al., 1981). Two $5 \mathrm{MHz}$ signals from iMaser72 and EFOS28 are connected to two identical frequency comparators as $f_{y 1}$ and $f_{y 2}$ respectively. The reference signal of $5 \mathrm{MHz}$ is connected to a signal splitter to produce two identical signals that are then connected to the frequency comparators as $f_{x}$.

The experiment was set to measure frequency phase differences of the signals for the time period of $100 \mathrm{~s}$ with an averaging number of 10 . Measurements were sampled at 10 nanoseconds resolution, maximum frequency difference and variance is set at $1 \times 10^{-08}$ as a limiting factor to exclude noise in the data. The Time Instance Meter (TIM) module of the frequency comparator produces two sets of phase measurements (1) as direct measurement $f_{y 1}, f_{y 2}$, and $f_{x} ;(2)$ as $f_{y 1 x}$, and $f_{y 2 x}$, which are calculated as

$$
f_{y 1 x}[H z]=1+\frac{k\left(f_{y 1}-f_{x}\right)}{f_{x}}, f_{y 2 x}[H z]=1+\frac{k\left(f_{y 2}-f_{x}\right)}{f_{x}},
$$

where $k\left(1 \times 10^{6}\right)$ is the multiplicative factor for the frequency noise.

\section{Results and Discussion}

The results for the two MASER clocks are plotted in Figure 4, and the derived parameters from the long-term data are summarised in Table 1. The raw data for the EFOS28 maser and the iMaser72 maser have a range of $0.1163 \mu$ s and $0.1945 \mu$ s respectively. In general, both maser clocks indicate smooth offset data with small fluctuations. This might be due to noise in the GNSS signal, maser instrumentation and thermal variations among other things that result in fluctuation as explained in Allan and Weiss (1980) and Dach et al., (2002). The second quadratic trend model Equation 5, gives a very good coefficient of determination $\left(\mathrm{R}^{2}\right)$ of 0.9958 for iMaser72 and it yielded $\mathrm{R}^{2}$ value of 0.9768 for EFOS25 maser clock. The coefficients of the trend models are given in Table 1. Standard errors and F values for the two masers are all very small and statistically significant, indicating good performance of the trend models. 

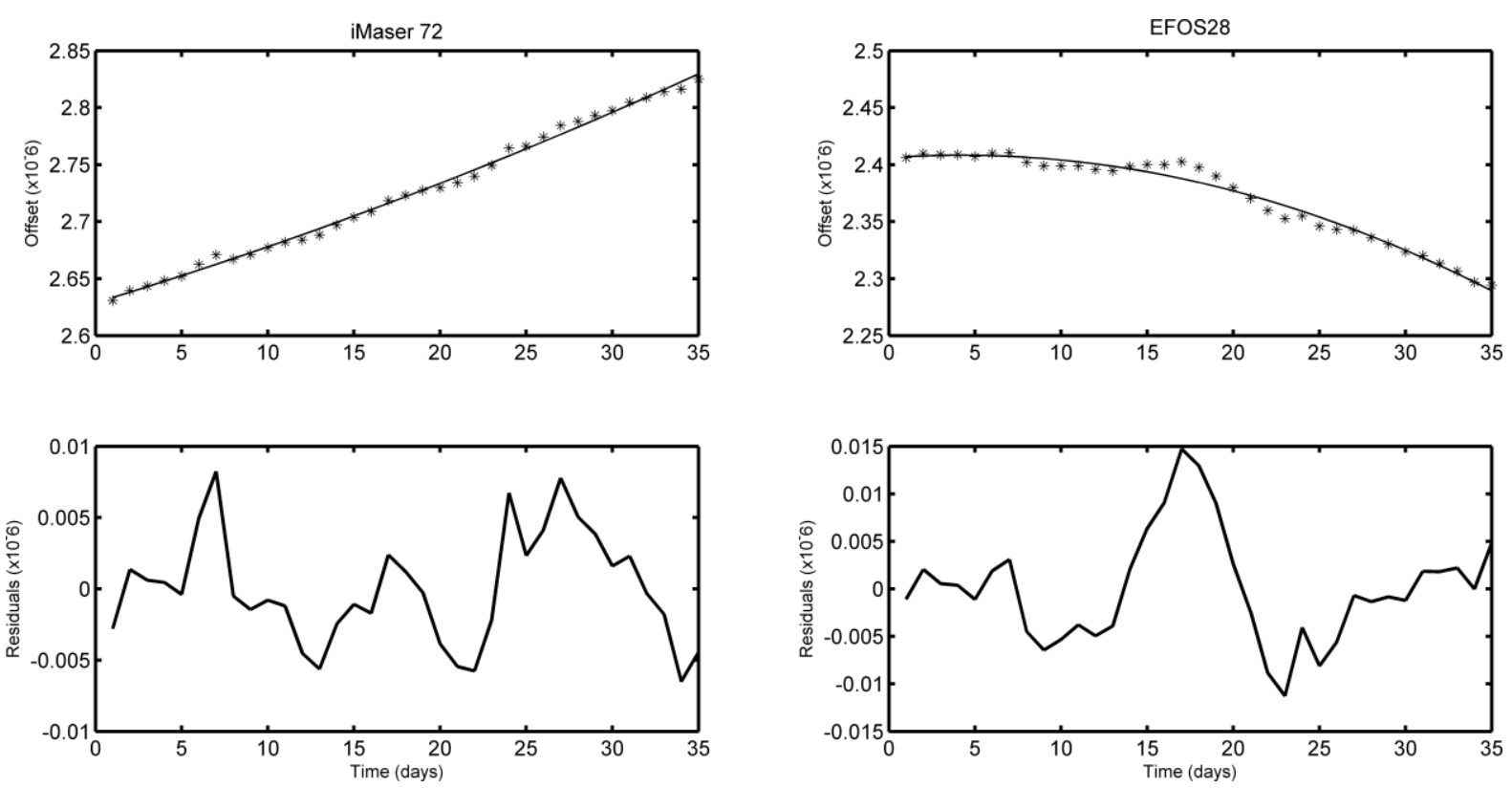

Figure 4. Time offset data for the EFOS28 and iMaser72 masers (top) and their least-squares residuals (bottom), (the units of the data in all the plots are in $\mu$ s).

The residual plots in Figure 4 indicate parameters that are not modelled. The standard deviation of the iMaser72 and EFOS28 residuals are 0.0053 and $0.0060 \mu \mathrm{s}$, respectively with a clear pattern. This pattern could be related to a number of factors which are (1) local environment where maser clocks are stored, especially thermal variations. The microwave cavity of the maser is very sensitive to shock-vibrations, atmospheric pressure, DC magnetic field and temperature fluctuations. A change of $1{ }^{\circ} \mathrm{C}$ of the microwave cavity can results in frequency change of about $3.5 \times 10^{-15}$ and this will result in fluctuation of the output frequency (Goujon et al., 2010). Hence it is important to keep the storage room at a constant temperature to minimise frequency fluctuations. (3) Radio signals transmitted by the GPS satellites to the receiving station are adversely affected by ionospheric delay (Allan and Weiss, 1980), tropospheric delay (Böhm et al., 2015), multipath and satellite clock errors. A good example to illustrate the effect of ionospheric effect on GPS applications (in this case, timing applications) is given by Allan and Weiss (1980), they estimated that a Total Electron Content of about $10^{18}$ electrons per metre squared will result in a delay of about $54 \mathrm{~ns}$ at low latitude at $1.575 \mathrm{GHz}$ C/A carrier frequency. This value can exceed $100 \mathrm{~ns}$ during periods of solar maximum. All these factors contribute towards the pattern that is evident on the residual plots.

Based on the observational data period used in this study, the calculated parameters indicate that both masers have similar performance. The fractional frequency errors are $-8.6554 \times 10^{-14}$ and $7.9063 \times 10^{-14}$ for the EFOS28 and iMaser72 maser respectively. An example is given to illustrate a practical use of the method described in section 4. By applying Equation 8, a value of $5751.686454 \mathrm{~Hz}$ must be applied to the EFOS28 maser to correct for the drift. The iMaser72 maser can be corrected by applying a value of $\Delta f$ directly 
to the maser. If $\Delta f$ is positive, a negative value of $\Delta f$ must be applied to turn the clock around and the opposite applies. Note that these masers are corrected differently because they were manufactured by different companies. Differences in drift rates can be attributed to many factors, such as different oscillator properties that are used to generate the $5 \mathrm{MHz}$ reference signal, thermal variations, supplied voltage fluctuations, crystal structure and impurities of the OCXO crystal or external factors that are discussed above (Prestage et al., 1995; Walsworth et al., 1990).

Table 1. Statistical and clock parameters as determined through least squares.

\begin{tabular}{lll}
\hline Statistical Parameters & EFOS28 (15 m) & iMaser72 (26 m) \\
\hline R Square & 0.9768 & 0.9958 \\
Standard Error $(\mu \mathrm{s})$ & 0.0059 & 0.0039 \\
y-Intercept & $2.4062 \pm 0.0032$ & $2.6289 \pm 0.0021$ \\
$b_{1}$ & $0.0011 \pm 0.0004$ & $0.00457 \pm 2.724 \times 10^{-4}$ \\
$b_{2}$ & $-0.0001 \pm 1.1013 \times 10^{-5}$ & $3.3227 \pm 7.339 \times 10^{-6}$ \\
Significance F-values & $7.28 \times 10^{-27}$ & $8.98 \times 10^{-39}$ \\
Calculated clock parameters & \\
Drift (s/d) & $-7.4783 \times 10^{-9}$ & $6.8310 \times 10^{-9}$ \\
Ageing (s/d/d) & $-2.5096 \times 10^{-10}$ & $6.6456 \times 10^{-11}$ \\
Fractional Frequency Error & $-8.6554 \times 10^{-14}$ & $7.9063 \times 10^{-14}$ \\
\hline
\end{tabular}

The individual short-term performances of the three maser clocks are depicted in Figure 5, where the iMaser72 has better performance over $1 \mathrm{~s}$ compared to the EFOS28 and EFOS6. Between 2 and $50 \mathrm{~s}$, the reference signal (EFOS6) seems to have better performance while other clocks have similar performance within this range. The derived fractional frequency error values (Table 1), are in good agreement with the short-term results.

In general, both masers perform at an expected level required for radio astronomy and geodetic VLBI applications. The highly accurate timing systems contribute towards VLBI system integrity and the accuracy of the observations (whereas factors such as mechanical errors, axis offset deformations due to temperature variations, etc. adversely affect system integrity) (Combrinck and Merry 1997). Hence, it is important for the observatories to keep track of their local timing systems to ensure that accurate observations are made. 


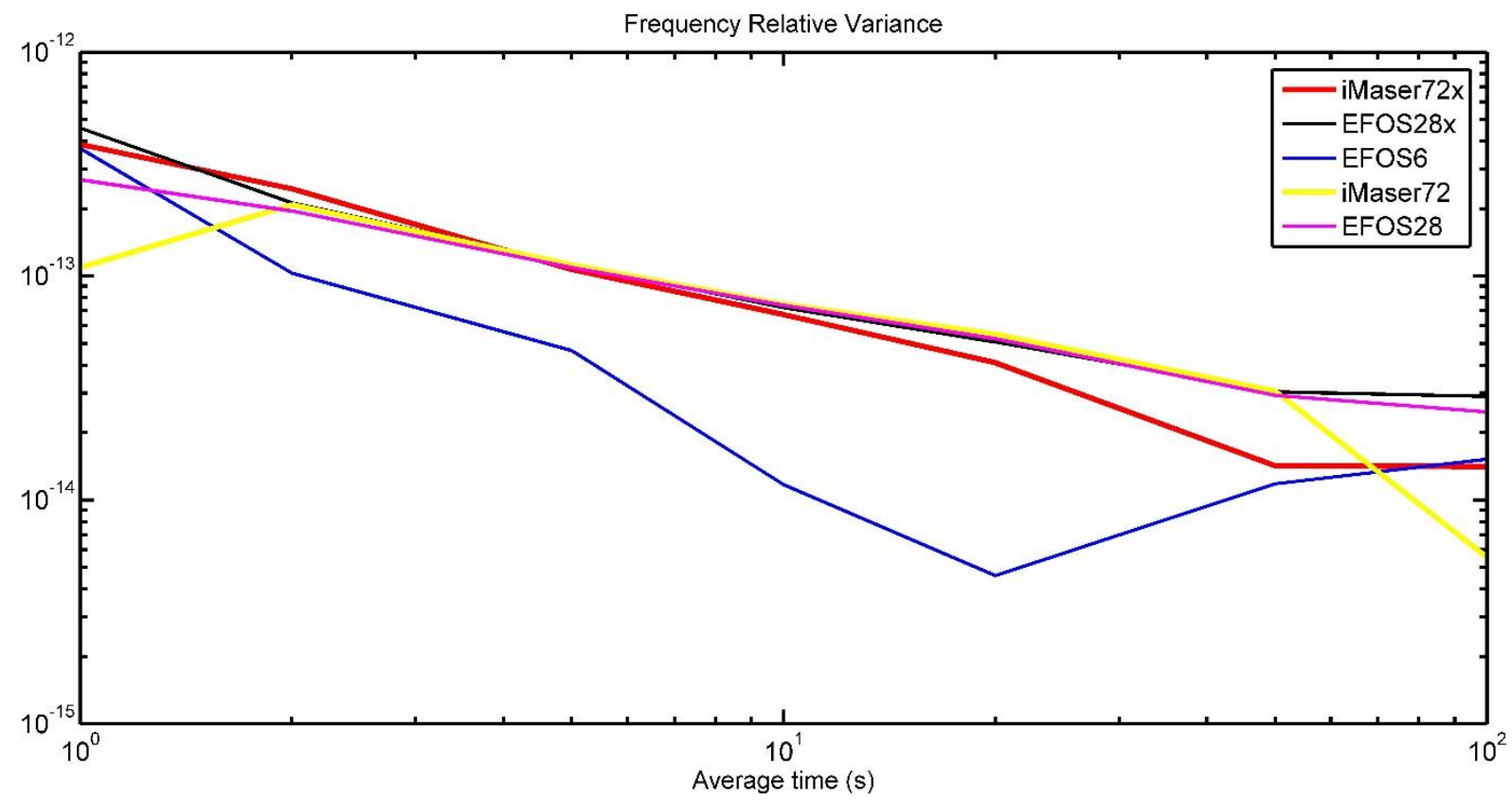

Figure 5. Individual frequency relative variance performance of each hydrogen maser clock.

\section{Conclusion}

The least-squares technique was used in this study to derive hydrogen maser clock parameters, enabling the management of maser clocks at HartRAO to function within predefined limits for VLBI applications. Small deviations that became apparent in the residuals require further investigations to determine the relationship between unmodeled components in the GNSS signal, which might be related to ionospheric or tropospheric effects. This could lead to the removal of the unexplained trend in the residuals. It was demonstrated that the two maser clocks currently used at HartRAO can maintain a relative stability of better than a picosecond $\left(10^{-12}\right)$, which is important to geodetic VLBI and radio astronomy applications and our results compare favourably with the specifications by the manufacturers. 


\section{Acknowledgements}

This research was financially supported by the National Research Foundation (NRF), the University of Pretoria and the Department of Science and Technology (DST). The authors would also like to thank the VLBI team at HartRAO for providing the data used in this study.

\section{References}

Allan DW 1987, 'Time and Frequency (Time-Domain) Characterization, Estimation, and Prediction of Precision Clocks and Oscillators', IEEE Transactions on Ultrasonics, Ferroelectrics, and Frequency Control, vol. UFFC-34, no. 6, pp. 647-654.

Allan DW \& Weiss MA 1980, 'Accurate time and frequency transfer during common-view of a GPS satellite', Proc. 43th Ann. Frequency Control Symposium, USAEADCOM, Ft. Monmouth, NJ 07703, National Bureau of Standards Boulder, Colorado, pp. 334-346.

Böhm J, Möller G, Schindelegger M, Pain G \& Weber R 2015, 'Development of an improved empirical model for slant delays in the troposphere (GPT2w) ', GPS Solutions, vol. 19, no. 3, pp. 433-441, doi: 10.1007/s10291-014-0403-7.

Combrinck L 2013, 'General Relativity and Space Geodesy', in Xu G (eds.), Sciences of Geodesy-II: Innovation and Future Developments. Spriger-Verlag, Berlin, pp. 53-95.

Combrinck L 2009, 'The MOBLAS-6 Satellite Laser Ranging Station at Hartebeesthoek, South Africa; Technology and Data Application', in Proceedings of the IEEE International Geoscience \& Remote Sensing Symposium, Earth observation, origins to applications, Cape Town, July 2009, pp. 161-164.

Combrinck WL \& Merry CL 1997, 'Very Long Baseline Interferometry antenna axis offset and intersections determination using GPS'. Journal Geophysical Research, vol. 102, no. B11, pp. 24, 741-24, 743.

Combrinck L 2014, 'Space geodesy, VLBI and the fourth pillar of geodesy; spacetime curvature', 8th IVS General Meeting "VGOS: The New VLBI Network" Shanghai (China), 2-7 March 2014, Shanghai, China.

Ciufolini I 1990, 'General relativistic measurements with satellite laser ranging, lunar laser ranging and very long baseline interferometry', Kluwer Academic Publishers, vol. 13, no. 1, pp. 67-78.

Chernyshev IN, Belyaev AA \& Mishagin KG 2012, 'Error in measurement of frequency instability by method of three oscillators', Measurements Techniques, vol. 55(7), pp. 792-799.

Dach R, Schilknecht T, Springer T, Dudle G \& Prost L 2002, 'Continuous Time Transfer Using GPS Carrier Phase', IEEE transactions on ultrasonics, ferroelectrics, and frequency control, vol. 49(1), pp. 1480-1490.

Donley EA, Heavner TP, Tataw MO, Levi F \& Jefferts SR 2004, 'Progress Towards the SecondGeneration Atomic Fountain Clock at NIST', Proceedings of the 2004 IEEE International Frequency Control Symposium and Exposition. Doi: 10.1109/FREQUATION2004.1573617.

Edwards C, Rogstad D, Fort D, White L \& Lijima B 1992, 'The Golden Real-Time Connected Element Interferometer', TDA Progress Report, pp. 42 -110. 
Ely T, Seubert J \& Bell J 2014, 'Advancing Navigation, Timing, and Science with the Deep Space Atomic Clock', SpaceOps 2014 13th International Conference on Space Operations, Pasadena, CA, American Institute of Aeronautics and Astronautics, May 2014.

Goujon D, Rochat P, Mosset P, Boving D, Perri A, Rochat J, Ramanan N, Simonet D, Vernez X \& Froidevaux S 2010, Development of the space active hydrogen maser for the aces mission, In EFTF-2010 $24^{\text {th }}$ European frequency and time forum. Institute of Electrical and Electronics Engineers, pp. 1-6.

Groslambert J, Fest D, Oliver M \& Gagnepain JJ 1981, 'Characterization of Frequency Fluctuations by Cross correlations and by Using Three or More Oscillators', Thirty Fifth Annual Frequency Control Symposium, Usaeradcom, Ft. Monmouth, NJ 07703, May 1981, Doi: 10.1109/FREQUATION1981.200512.

Levine DM, Berenson ML \& Stephan D 1999, 'Statistics for Managers Using Microsoft Excel', Prentice-Hall, New Jersey.

Levine MW \& Vessot RFC 1970, 'Hydrogen-Maser Time and Frequency Standard at Agassiz Observatory', Radio Science, vol. 5, no. 10, pp. 1287-1292.

Lewandowski W, Azoubib J \& Klepczynski WJ 1999, 'GPS: Primary Tool for Time Transfer', Proceedings of the IEEE, vol. 87, no. 1.

Lemke ND, Ludlow AD, Barber ZW, Fortier TM, Diddams SA, Jiang Y, Jefferts SR, Heavner TP, Parker TE \& Oates CW 2009, 'Spin-1/2 Optical Lattice Clock', PRL, 103, 063001. doi: 10.1103/PhysRevLett.103.063001.

Lombardi MA 2002, 'Chapter 17: Fundamentals of time and frequency', in Bishop RH (eds.), The Mechatronics Handbook, CRC Press.

Mayer D, Böhm J, Combrinck L, Botai J \& Böhm S 2014, 'Importance of the Hartebeesthoek Radio Astronomy Observatory for the VLBI network', Acta Geodaetica et Geophysica, vol. 49,no. 3, pp. 313-325, doi:10.1007/s40328-014-0063-7.

Mizuhiko H 2003, 'Atomic Frequency Standards: Basic Physics in the Atomic Frequency Standards', Journal of the National Institute of Information and Communications Technology, vol. 50, no. 1/2.

Middelberg E \& Bach U 2008, 'High resolution radio astronomy using very long baseline interferometry', Reports on Progress in Physics, vol. 71, no. 6, doi:10.1088/00344885/71/6/066901.

Moran J 1989, 'Introduction to VLBI', in Felli M and Spencer RE (eds.), Very long baseline interferometry, techniques and applications. Kluwer Academic Publishers, Dordrecht, pp: 27-45

Niell A, Whitney A, Petrachenko B, Schlüter W, Vandenberg N, Hase H, Koyama Y, Ma C, Schuh H \& Tuccari G 2005, 'VLBI2010: Current and Future Requirements for Geodetic VLBI Systems. Report of Working Group 3 to the IVS Directing Board, Technical report, viewed 12 June 2015, $<\mathrm{ftp} / / / 152.74 .113 .3 /$ radiotelescopio/IVS_WG3report.pdf $>$.

Petrachenko B, Niell A, Behrend D, Corey B, Böhm J, Charlot P, Collioud A, Gipson J, Haas R, Hobiger T, Koyama Y, MacMillan D, Malkin Z, Nilsson T, Pany A, Tuccari G, Whitney A \& Wresnik J 2009, 'Design aspects of the VLBI2010 system', Progress report of the IVS VLB I2010 committee, Technical report, viewed 02 September 2015, <http://adsabs.harvard.edu/ abs/ 2009vlbi.rept....1P>.

Plag H-P, Altamimi Z, Bettadpur S, Beutler G, Beyerle G, Cazenve A, Crossley D, Donnellan A, Forsberg R, Gross R, Hinderer J, Komjathy A, Ma C, Mannucci AJ, Noll C, Nothnagel A, Pavlis EC, Pearlman M, Poli P, Schreiber K, Senior K, Woodworth PL, Zerbini S \& Zuffada C 2009, 'The goals, achievements, and tools of modern geodesy'. In: Plag HP \& Pearlman M (eds.), Global 
Geodetic Observing System: Meeting the Requirements of a Global Society on Changing Planet in 2020. Berlin Heidelberg, Springer-Verlag, pp. 15-87.

Prestage JD, Tjoelker RL \& Maleki L 1995, 'Atomic clocks and variations of the fine structure constant', Physical Review Letters, vol. 74, no. 18, pp. 3511-3514.

Robertson DS 1991, 'Geophysical applications of very-long-baseline interferometry', Reviews of Modern Physics, vol. 63, no. 4, pp. 899-918.

Ronnang BO 1989, 'Geodesy, Geodynamics, and Astrometry Using Very Long Baseline Interferometry', in Felli M and Spencer R (eds.), Very Long Baseline Interferometry, Techniques and Applications, NATO ASI Series, Springer Netherlands, vol. 283, pp. 305-317.

Schuh H \& Böhm J 2012, 'Very Long Baseline Interferometry for Geodesy and Astrometry', in Xu G (eds.), Sciences of Geodesy II, Springer Berlin Heidelberg, pp. 339-376.

Schuh H \& Heinkelmann R 2010, 'Very long baseline interferometry: accuracy limits and relativistic tests', in Klioner SA, Seidelman PK and Soffel MH (eds.), Relativity in Fundamental Astronomy, Proceedings IAU symposium, No. 261. doi:10.1017/S1743921309990524

Schuh H \& Behrend D 2012, 'VLBI: A fascinating technique for geodesy and astrometry', Journal of Geodynamics, vol. 61, pp. 68-80.

Seeber G 1993, 'Satellite Geodesy', $1^{\text {st }}$ edition, Walter de Gruyter, Berlin.

Seubert J \& Ely TA 2015, 'One-Way Radiometric Navigation with the Deep Space Atomic Clock', California Institute of Technology, AAS/AIAA Space Flight Mechanics Meeting, At Williamsburg, VA, pp. 15-384.

Vessot RFC 2005, 'The atomic hydrogen maser oscillator', Istitute of Physics Publishing, Metrologia, vol. 42, no. S80-S89.

Wei-qun Z, Chuan-fu L, Shuang-lin Y, Guan-zhong W, Yi-ping Z, Pei-hong Y \& Jian Z 2001 'A study and performance evaluation of hydrogen maser used in Chinese mobile VLBI stations', Chin Astron Astrophys, vol. 25, no. 3, pp. 390-397.

Walsworth RL, Silvera IF, Mattison EM \& Vessot RF 1990, 'Test of the linearity of quantum mechanics in an atomic system with a hydrogen maser', Physical review letters, vol. 64. no. 22, pp. 2599-2602.

Wynands R \& Weyers S 2005, 'Atomic fountain clocks', Metrologia, vol. 45, doi:10.1088/00261394/42/3/S08. 